\title{
Predicting spike timing of neocortical pyramidal neurons by simple threshold models
}

\author{
Renaud Jolivet • Alexander Rauch • \\ Hans-Rudolf Lüscher • Wulfram Gerstner
}

Received: 26 September 2005 / Revised: 21 December 2005 / Accepted: 11 January 2006 / Published online: 22 April 2006

(C) Springer Science + Business Media, LLC 2006

\begin{abstract}
Neurons generate spikes reliably with millisecond precision if driven by a fluctuating current-is it then possible to predict the spike timing knowing the input? We determined parameters of an adapting threshold model using data recorded in vitro from 24 layer 5 pyramidal neurons from rat somatosensory cortex, stimulated intracellularly by a fluctuating current simulating synaptic bombardment in vivo. The model generates output spikes whenever the membrane voltage (a filtered version of the input current) reaches a dynamic threshold. We find that for input currents with large fluctuation amplitude, up to $75 \%$ of the spike times can be predicted with a precision of $\pm 2 \mathrm{~ms}$. Some of the intrinsic neuronal unreliability can be accounted for by a noisy threshold mechanism. Our results suggest that, under random current injection into the soma, (i) neuronal behavior in the subthreshold regime can be well approximated by a simple linear filter; and (ii) most of the nonlinearities are captured by a simple threshold process.
\end{abstract}

\section{Action Editor: Matthew Wiener}

R. Jolivet $(\triangle) \cdot$ W. Gerstner

Ecol Polytechnique Federale de Lausanne (EPFL),

School of Computer and Communication Sciences and Brain

Mind Institute, Station 15,

CH-1015 Lausanne, Switzerland

e-mail: renuad.jolivet@epfl.ch

W. Gerstner

e-mail: wulfram.gerstner@epfl.ch

\section{A. Rauch}

University of Bern, Institute of Physiology,

CH-3012 Bern, Switzerland; Max Planck Institute for Biological

Cybernetics, D-72012, Tübingen, Germany

H.-R. Lüscher

University of Bern, Institute of Physiology,

CH-3012 Bern, Switzerland
Keywords Spike Response Model - Stochastic input . Adapting threshold $\cdot$ Spike-timing reliability $\cdot$ Predicting spike timing.

\section{Introduction}

Informally, neuroscientists often talk about neurons as units that sum up the postsynaptic potentials caused by presynaptic spike arrival until a threshold of spike generation is reached. This is also the level of description used in minimal models such as the Integrate-and-Fire or Spike Response Model (Lapicque, 1907; Hill, 1936; Stein, 1967; Gerstner and Kistler, 2002). On the other hand, it is well known that dendritic processes are highly non-linear (Stuart and Sakmann, 1994; Helmchen et al., 1999; Larkum et al., 2001) and that spike generation cannot be accounted for by a simple voltage or current threshold (Koch et al., 1995; FourcaudTrocmé et al., 2003).

While minimal models cannot describe neuronal activity for all different types of artificial stimuli that can be applied in elaborate experimental paradigms, a minimal model should ideally be capable of predicting neuronal spiking under those conditions that are potentially relevant for a neuron in vivo. A typical cortical neuron receives input from thousand of other cortical neurons (Braitenberg and Schütz, 1991) as well as input from subcortical areas such as the thalamus. It is an open issue, how much of this synaptic bombardment can be thought of as random or as a potentially meaningful signal. Rapidly varying visual inputs can fire motion-sensitive cortical neurons with high temporal precision (Bair and Koch, 1996; Buracas et al., 1998) which suggests that neurons receive temporally precise stimulus-locked signals. In both visual cortex (Borg-Graham et al., 1998) and auditory cortex (Wehr and Zador, 2003) input signals comprise excitatory 
and inhibitory components. Despite the temporal precision under appropriate stimulation conditions, neuronal responses can be highly variable across trials, in particular in visual cortex (Heggelund and Albus, 1978; Buracas et al., 1998), but much less so in auditory cortex (De Weese and Zador, 2003) or the retina (Berry et al., 1997). The major source of this variability in spike count seems to be a partially correlated background input from other parts of the cortical network (Gawne and Richmond, 1993; Arieli et al., 1996; Bair et al., 2001; Steriade et al., 2001; Destexhe et al., 2003; De Weese and Zador, 2004). Correlations in the background input could occur on a slow (Arieli et al., 1996; Steriade et al., 2001) or a much faster time scale of only a few milliseconds (Bair et al., 2001). One hypothetical interpretation of the irregular firing of cortical neurons is that background activity of the cortical network provides brief but strongly synchronized input bursts at times that are not locked to any stimulus (Abeles, 1991; Stevens and Zador, 1998; Diesmann et al., 1999; De Weese and Zador, 2004).

Since the statistics of the input a cortical neuron would receive in vivo depends on the area of cortex and is only partially known see, e.g., Borg-Graham et al., 1998; Steriade et al., 2001; Wehr and Zador, 2003, we adopted a minimal approach. Similar to previous work (Bryant and Segundo, 1976; Mainen and Sejnowski, 1995) we stimulated 24 neocortical pyramidal neurons in vitro with a time-dependent current while recording the neuronal membrane potential under patch clamp (Rauch et al., 2003). The very same fluctuating current that is used in the experiments is also applied to the simple threshold model. This highly fluctuating input current is supposed to model the total input a neuron would receive, i.e., random as well as correlated spike arrival. Even though it is generated by a random process, we do not think of the input as "noise", but rather as a rapidly fluctuating temporal signal.

In contrast to more traditional methods of model evaluation which mainly focus on mean firing rates and, potentially, interspike interval distributions (Tuckwell, 1988; Bugmann et al., 1997; Troyer and Miller, 1997; Rauch et al., 2003; La Camera et al., 2004), we assess the quality of our threshold model on a spike-by-spike basis. More precisely, we classify an output of the model neuron as correct only if the action potential of the model occurs within \pm 2 ms of that of the pyramidal cell. Surprisingly, we find that for input currents with large fluctuation amplitude, up to $75 \%$ of the action potentials in the pyramidal cell can be predicted by the simple threshold model.

While we do not address questions of neuronal coding per se (Abeles, 1991; Bialek et al., 1991; Theunissen and Miller, 1995; Rieke et al., 1996) our approach, based on a comparison of models with data at a millisecond resolution, is inspired by experiments that have measured the temporal precision of neuronal activity (Bair and Koch, 1996; Berry et al., 1997; de Ruyter van Stevenick et al., 1997; Buracas et al., 1998; Ikegaya et al., 2004; Johansson and Birznieks, 2004). In particular, for single neurons in vitro, it is well known that the intrinsic reliability of neuronal spike timing on a millisecond time scale is highest if the input current has a large fluctuation amplitude, but much lower when the input is constant or shows small fluctuations only (Mainen and Sejnowski, 1995). We will show in this paper that a completely analogous statement holds for the quality of our simple threshold model: The capability of the model in predicting the exact timing of neuronal action potentials is good for largely fluctuating currents, but drops for small fluctuations of the input current.

In this paper, we apply a method of rapid parameter extraction (Jolivet et al., 2004) in order to show that a simple threshold model can explain up to $70 \%$ of the spike times of a pyramidal neuron under random current injection.

The paper is organized as follows. We start with a simple version of our threshold model and asses its quality by comparing the model output with that of the pyramidal cells in terms of neuronal firing times and subthreshold voltage traces. As we will see the model works best if an adaptation mechanism that automatically adjusts the firing threshold is included. Our basic threshold model is then extended. We account for neuronal unreliability by a phenomenological model of intrinsic noise sources. Details of the model and of the numerical procedures can be found in the Materials \& methods section.

\section{Materials and methods}

\subsection{Experimental preparation and recordings}

Parasagittal slices of rat somatosensory cortex $(300 \mu \mathrm{m}$ thick) were prepared from 15- to 40-day-old female and male Wistar rats according to the institutional guidelines. We recorded in current-clamp whole cell configuration from the soma of layer 5 regular spiking pyramidal cells (McCormick et al., 1985). 20 cells are part of the dataset presented by Rauch and colleagues (2003). We included 4 additional cells recorded in the same setup where trial repetitions of the input were performed ( $N=24$ cells in total). See (Rauch et al., 2003) for further details about the recording protocol and the setup.

For the stimulation, we assume that a large number of excitatory and inhibitory presynaptic neurons emit spikes at random times. On the postsynaptic side this heavy barrage is modeled as a total synaptic current $I$ that fluctuates randomly around some mean value $\mu_{I}$. If the contribution of each synaptic input is small, the input can be described as an Ornstein-Uhlenbeck process (Tuckwell, 1988). For reasons of mathematical simplicity, we assume that the 
time courses of AMPA and $\mathrm{GABA}_{\mathrm{A}}$ receptors are the same $\left(\tau_{\text {ampa }}=\tau_{\text {gaba }}=\tau_{I}\right)$ with a zero rise time to the peak current which represents a coarse description of biological reality (Häusser and Roth, 1997). The advantage of this simplification is that the total synaptic current $I(t)$ can be generated with a single equation

$I(t+d t)=I(t)-\frac{I(t)}{\tau_{I}} d t+m_{I} d t+s_{I} \xi(t) \sqrt{d t}$

where $m_{I}$ and $s_{I}$ are parameters and $\xi(t)$ is a unitary Gauss distributed random variable, updated at every time step. The process was generated and injected at a rate of $5 \mathrm{kHz}(d t=0.2 \mathrm{~ms})$ and the correlation length $\tau_{I}$ was $1 \mathrm{~ms}$. The resulting current $I(t)$ has a stationary Gaussian distribution with mean $\mu_{I}=m_{I} \tau_{I}$ and variance $\sigma_{I}^{2}=s_{I}^{2} \tau_{I / 2}$ (Cox and Miller, 1965).

The parameters $\mu_{I}$ and $\sigma_{I}$ were systematically varied as follows: the total range $0<\mu_{I}<900 \mathrm{pA}$ and $0<\sigma_{I}<700$ $\mathrm{pA}$ was discretized and then explored in random order to prevent correlations over time. The duration of the stimulation was between 10-30 s long for a given couple of parameters $\mu_{I}, \sigma_{I}$. The first transient part of the neuronal response ( $1 \mathrm{~s}$ ) was discarded for the analysis presented in the Results section. The intervals between successive stimulations were 50-60 s long. Particular care was taken to ensure that the response of the cell was consistent throughout the whole recording session. Usually the cells showed a transient phase at the beginning, followed by a long time interval (10$90 \mathrm{~min}$ ) during which the response was consistent (when the same current was injected, the average spike frequency did not change significantly), and by a final unstable phase. Cells with a stable period shorter than 40 min were not included in the analysis (see Rauch et al. (2003) for further details).

\subsection{Model of neuronal activity}

The Spike Response Model (Kistler et al., 1997; Gerstner and Kistler, 2002) is a generalization of the classic Integrateand-Fire model. In this framework, the state of a neuron is described by a single equation for the membrane voltage plus a threshold condition for spike emission. The membrane potential $u$ of the cell at time $t$ is given by

$u(t)=\eta(t-\hat{t})+\int_{0}^{+\infty} \kappa_{\infty}(s) I^{\mathrm{ext}}(t-s) d s$

for $t>\hat{t}$ where $\hat{t}$ denotes the timing of the last spike emitted by the model neuron. The last term in Eq. (2) accounts for the external driving current $I^{\mathrm{ext}}(t)$. The input integration process is characterized by the function $\kappa_{\infty}$. The function $\eta$ includes the form of the spike itself as well as a depolarizing or hyperpolarizing afterpotential. An absolute refractory period $\gamma_{\text {ref }}=2 \mathrm{~ms}$ is used in simulations to avoid spiking immediately after a first spike has been triggered. A spike is elicited if the following threshold condition is satisfied

if $u(t) \geq \vartheta(t) \quad$ and $\quad \frac{d u}{d t}(t) \geq \frac{d \vartheta}{d t}(t) \quad$ then, $\hat{t}=t$

Note that spiking occurs only if the membrane voltage crosses the threshold $\vartheta$ from below. The firing time $\hat{t}$ plays the role of a reset variable. This is different from the Integrate-and-Fire model where the voltage is reset after a spike. The threshold itself can be taken either as a constant, as time-dependent or as adapting. In this paper, we use an adapting threshold. Each time that a spike is emitted by the model, the threshold $\vartheta$ is increased by a given amount $\vartheta \rightarrow \vartheta+A_{\vartheta}$. Between spikes, $\vartheta$ decays back to a resting value $\vartheta_{0}^{A}$ with time constant $\tau_{\vartheta}^{A}$

$\frac{d \vartheta}{d t}=-\frac{\vartheta-\vartheta_{0}^{A}}{\tau_{\vartheta}^{A}}+A_{\vartheta} \sum_{k} \delta\left(t-t_{k}\right)$

where $t_{k}$ is the timing of outgoing spikes. It follows from Eq. (4) that, if the frequency is much larger than $1 / \tau_{\vartheta}^{A}$, the average threshold $\bar{\vartheta}$ depends linearly on the output frequency $f$

$\bar{\vartheta} \approx \vartheta_{0}^{A}+\alpha f$

with $\alpha=A_{\vartheta} \tau_{\vartheta}^{A}$. This type of adaptation mechanism has been shown to constitute a universal model for spike-frequency adaptation (Benda and Herz, 2003) and has already been applied in a similar context (La Camera et al., 2004). During the model estimation, we use as a first step a traditional constant threshold denoted by $\vartheta(t)=\vartheta_{\text {cst }}$ which is then transformed in the adaptive threshold of Eq. (4) by a procedure to be detailed below.

The specific version of the Spike Response Model that we use in this paper, defined by Eqs. (2) and (3), has been termed $\mathrm{SRM}_{0}$ (Gerstner and Kistler, 2002) but for the sake of simplicity, we will simply use Spike Response Model (or $\mathrm{SRM}$ ) to refer to it. In the full version of the Spike Response Model, the function $\kappa$ is made time-dependent, i.e. it also depends on $t-\hat{t}$. This dependence accounts for the fact that the response of a neuron, and more specifically the effective membrane time-constant, is usually reduced shortly after an emitted spike (Fuortes and Mantegazzini, 1962; Powers and Binder, 1996; Stevens and Zador, 1998). This dependence is of importance to account for spike-to-spike interactions (Arcas and Fairhall, 2003). The mapping procedure that we discuss in the following allows, in principle, to compute $\kappa(t-\hat{t} ; s)$ for any $t-\hat{t}$ (see Jolivet et al.,2004). However, it requires longer recordings than the ones provided by typical experiments. The critical factors are the number of 
spikes and the average time interval between two consecutive spikes. The mapping procedure needs ideally both as many spikes as possible and long intervals. We therefore decided to restrict ourselves to the simple version of the Spike Response Model defined by Eqs. (2) and (3). Finally, note that this model is closely related to the models proposed by Keat and colleagues (2001) and Paninski and colleagues (2005).

\subsection{Evaluation of performances}

The coincidence factor $\Gamma$ between two spike trains (Kistler et al., 1997) is defined by

$\Gamma=\frac{N_{\text {coinc }}-\left\langle N_{\text {coinc }}\right\rangle}{\frac{1}{2}\left(N_{\text {data }}+N_{\text {SRM }}\right)} \frac{1}{N}$

where $N_{\text {data }}$ is the number of spikes in the reference spike train $S_{\text {data }}, N_{\mathrm{SRM}}$ is the number of spikes in the predicted spike train $S_{\mathrm{SRM}}$ that is compared with the reference spike train, $N_{\text {coinc }}$ is the number of coincidences with precision $\Delta$ between the two spike trains, and $\left\langle N_{\text {coinc }}\right\rangle=2 v \Delta N_{\text {data }}$ is the expected number of coincidences generated by a homogeneous Poisson process with the same rate $v$ as the spike train $S_{\mathrm{SRM}}$. In this paper, the reference spike train $S_{\text {data }}$ consists of the recordings of pyramidal cells in vitro while $S_{\mathrm{SRM}}$ is the spike train generated by the SRM with the same driving current. The factor $N=1-2 v \Delta$ normalizes $\Gamma$ to a maximum value of 1 which is reached if and only if the spike train of the SRM reproduces exactly that of the cell. A homogeneous Poisson process with the same number of spikes as the SRM would yield $\Gamma=0$. We compute the coincidence factor $\Gamma$ by comparing the two complete spike trains, i.e., the recorded spike train $S_{\text {data }}$ and the train $S_{\text {SRM }}$ predicted by the SRM as in Jolivet et al. (2004). Therefore, in this paper, $\Gamma$ provides a measure of the ability of the minimal model to predict the full spike train of a cortical neuron. Throughout the paper, we use $\Delta=2 \mathrm{~ms}$. Results do depend on $\Delta$ but the exact value of $\Delta$ is not critical as long as it is chosen in a reasonable range $1 \leq \Delta \leq 4 \mathrm{~ms}$ (Jolivet, 2005). Note that the coincidence factor $\Gamma$ is similar to the "reliability" as defined in Mainen and Sejnowski (1995). All measures of $\Gamma$ reported in this paper are given for new stimuli, independent of those used for parameter optimization during the mapping procedure.

\subsection{Mapping procedure}

The technique used in this paper for extracting model parameters has been discussed in detail elsewhere (Jolivet and Gerstner, 2004; Jolivet et al., 2004; Jolivet, 2005) and we refer interested readers to these references. Here, we describe the essentials of the technique without going into details.
In short, we use a systematic step-by-step evaluation and optimization procedure based on intracellular recordings. It consists in sequentially evaluating the kernels $\left[\eta\right.$ and $\kappa_{\infty}$; Eq. (2) $]$ and parameters $\left[A_{\vartheta}, \vartheta_{0}^{A}\right.$ and $\tau_{\vartheta}^{A}$; Eq. (4) $]$ that characterize a specific instance of the model. The consecutive steps of the procedure are as follows:

1. Extract the kernel $\eta$ from a sample voltage recording by spike triggered averaging. We use a recording with a mean drive $\mu_{I}=0$ and keep the resulting $\eta$ fixed throughout the rest of the analysis.

2. Subtract $\eta$ from the voltage recording to isolate the subthreshold fluctuations.

3. Extract the kernel $\kappa_{\infty}$ by the Wiener-Hopf optimal filtering technique (Wiener, 1958; Lee and Schetzen, 1965; Jolivet et al., 2004). This step involves a comparison between the subthreshold fluctuations and the corresponding input current.

4. Find the optimal constant threshold $\vartheta_{\text {cst }}$. The optimal value of $\vartheta_{\text {cst }}$ is the one that maximizes the coefficient $\Gamma$. The parameter $\vartheta_{\text {cst }}$ depends on the specific set of input parameters (mean $\mu_{I}$ and variance $\sigma_{I}^{2}$ ) used during stimulation.

5. Plot the threshold $\vartheta_{\text {cst }}$ as a function of the firing frequency $f$ of the neuron and run a linear regression. $\vartheta_{0}^{A}$ is identified with the value of the fit at $f=0$ and $\alpha$ with the slope Eq. (5).

6. Optimize $A_{\vartheta}$ for the best performances (again measured with $\Gamma), \tau_{\vartheta}^{A}$ is defined as $\alpha / A_{\vartheta}$.

Each cell of the experimental dataset is characterized by it's own set of parameters $\eta, \kappa_{\infty}, \vartheta_{0}^{A}, A_{\vartheta}$ and $\tau_{\vartheta}^{A}$.

In the final part of the paper, the deterministic threshold model is transformed into a stochastic model by turning the strict threshold condition into a stochastic firing criterion. In other words, the strict condition for spike emission is replaced by a voltage-dependent instantaneous probability of firing $f(u-\vartheta)$ or stochastic intensity. A reasonable choice is

$f(u-\vartheta)=\frac{1}{\tau_{s}} \exp [(u-\vartheta) / \delta u]$

with $\vartheta$ the threshold, $\delta u$ the width of the spike emission zone and $\tau_{s}$ the mean time to spike emission at threshold. To maintain a direct relation with the deterministic model, $\vartheta$ is simply identified with the adapting threshold introduced in Eq. (4). To evaluate the two other parameters $\tau_{s}$ and $\delta u$, we estimate from the histogram of $(u-\vartheta)$ the joint density $p(u-\vartheta$, spike $)$ at the real neuron's firing times as well as the marginal distribution $p(u-\vartheta)$ where $(u-\vartheta)$ is calculated from our minimal model. By comparing these two histograms, i.e. by taking for each voltage difference the ratio of the number of instances with action potential triggered to the total number of instances with that voltage, it is possible 
to estimate the probability of firing in a time bin $d t$ given the voltage to threshold $(u-\vartheta)$ (Arcas et al., 2003). It can be shown that, due to time discretization, this probability is connected to $f(u-\vartheta)$ by Gerstner and Kistler (2002).

$\operatorname{Prob}\{$ spike in $[t, t+d t]\}=1-\exp (-d t f(u-\vartheta))$

For numerical evaluation, we only consider data where $d t f(u) \leq 0.3$ and use the Taylor approximation $1-$ $\exp (-d t f(u-\vartheta)) \approx d t f(u-\vartheta)-\frac{d t^{2}}{2} f(u-\vartheta)^{2}$. From this, we can extract numerical values for $\delta u$ and $\tau_{s}$ by a simple fitting procedure. Finally, the probability of the stochastic model to fire in coincidence with the real neuron, i.e. during an interval $\hat{t}-\Delta, \hat{t}+\Delta$ around the neuron's firing time $\hat{t}$, is given by

$$
\begin{aligned}
& \operatorname{Prob}\{\text { spike } \operatorname{in}[\hat{t}-\Delta, \hat{t}+\Delta]\} \\
& \quad=1-\exp \left(-d t \sum_{i=\hat{t}-\Delta}^{\hat{t}+\Delta} f\left(u_{i}-\vartheta_{i}\right)\right)
\end{aligned}
$$

which is used for the histogram of Fig. 6. See legend and corresponding text for further details.

\subsection{Numerics and statistics}

Ordinary differential equations were simulated with the forward Euler method with a time step $d t=0.2 \mathrm{~ms}$. Since this time step is much shorter than all other time constants of the minimal model, such a simple first-order method is sufficient.

Detection of spikes in experimental recordings was realized using a standard (Azouz and Gray, 2000) threshold condition on the first derivative of the membrane voltage (crossing from below). The exact value of this threshold is arbitrarily chosen but we ensure that it is as low as possible with zero false positives on a sample spike train of $30 \mathrm{~s}$ in order to be as close as possible to the spike initiation point. The voltage at the initiation point of a spike is defined as the effective threshold of this spike and is used in Fig. 5A.

Cross-correlation coefficients were measured in the subthreshold regime after removal of $4 \mathrm{~ms}$ during spikes. Crosscorrelation coefficients between PSTHs (Fig. 7) were measured on complete sequences.

For the Z-test in Fig. 3A, we used the following approach. All distributions in Fig. 3A were constructed using the same proportion of points in highly reliable $(50 \%)$ and intermediate regimes $(50 \%)$ as defined in the Results section. We assume that the distribution for cells without repetitions approximates the marginal distribution since it is constructed from many individual samples $(N=186$ samples $)$ while the distribution for cells with repetitions is constructed from a smaller ensemble ( $N=24$ samples). Given these pre- liminaries, it can be shown that the small sample, i.e. the distribution of $\Gamma_{m \rightarrow n}$ for cells with repetitions, originates from the marginal distribution with high probability (Z-test with 0.01 significance level).

\section{Results}

The results are organized as follows. In the following subsection (Deterministic model of neuronal activity), we map a simple deterministic threshold model to in vitro recordings of cortical neurons and explore the performance of that model in terms of spike timing prediction. The performance of the model is compared to the intrinsic reliability of cortical neurons. In the second subsection (Spike train variability and stochastic model of neuronal activity), we study the variability of spike trains. To account for this variability, we turn our deterministic model into a stochastic one by replacing the strict threshold by a firing probability and, again, compare the spike trains predicted by such a model to those of real neurons.

\subsection{Deterministic model of neuronal activity}

We recorded the activity of regularly spiking pyramidal cells in response to noisy current injection with stationary statistics. These cells constitute our set of so-called target neurons $(N=24)$. We then built for each recorded cell an effective minimal model that is able to yield quantitative predictions about the spike train of the cell.

The minimal model considered in this paper is the Spike Response Model (Kistler et al., 1997; Gerstner and Kistler, 2002). Just as in Integrate-and-Fire models (Stein, 1967), a spike is emitted only if the membrane voltage crosses a predetermined threshold. Threshold crossing defines the firing time $\hat{t}$. The basic assumption of the model is that the subthreshold dynamics during random current injection can be approximated by some linear filter $\kappa_{\infty}$. The nonlinearity of spike generation is reduced to a threshold process. The form of an action potential is given by the spike shape $\eta$. The membrane voltage after a spike triggered at time $\hat{t}$ is

$u(t)=\eta(t-\hat{t})+\int_{0}^{+\infty} \kappa_{\infty}(s) I^{\mathrm{ext}}(t-s) d s$

where $u(t)$ is the membrane voltage at time $t>\hat{t}$. The integral with the filter $\kappa_{\infty}$ accounts for the membrane polarization generated by the external driving current $I^{\mathrm{ext}}(t)$. Note that $\hat{t}$ always denotes the last firing time.

In order to map the model to in vitro recordings, we first need to find an expression for the functions $\eta$ and $\kappa_{\infty}$. The function $\eta$ is extracted from the data by smoothing the aver- 
Fig. 1 Functions $\eta$ (A) and $\kappa_{\infty}$ (B) as extracted by the method exposed in this paper. Raw data (symbols) and fit by double exponential functions (solid line). C. The optimal constant threshold $\theta_{\text {cst }}$ is plotted versus the output frequency $f$ (symbols). It is very neatly fitted by a linear function (line)
A

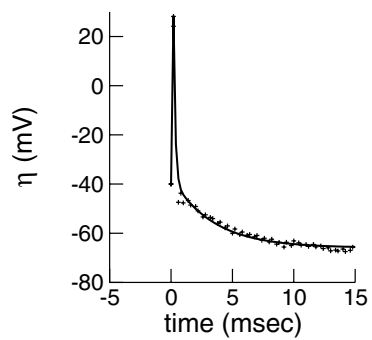

B

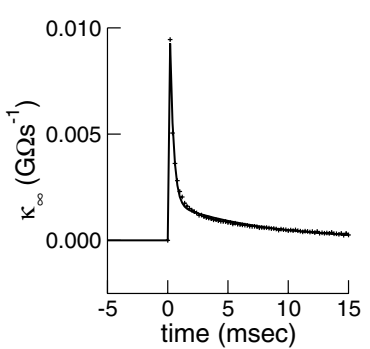

C

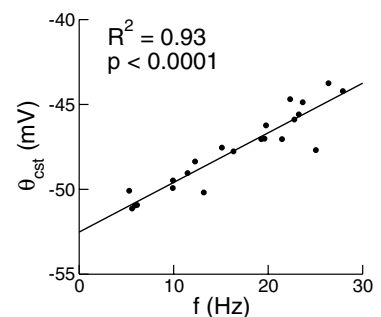

age shape of spikes when the mean drive is null and $\kappa_{\infty}$ is the best linear filter to account for the subthreshold variations of the membrane potential (Wiener, 1958; Lee and Schetzen, 1965; Jolivet and Gerstner, 2004; Jolivet et al., 2004). It is important to note that both $\eta$ and $\kappa_{\infty}$ are directly extracted from sample recordings, no approximation techniques are used beyond simple smoothing. Figure 1 shows functions $\eta$ and $\kappa_{\infty}$ for a given cell. In this specific case, the cell does not display afterhyperpolarization after emission of a spike and the voltage returns to the resting potential from above (Fig. 1A). The maximum of the action potential occurs about $0.5 \mathrm{~ms}$ after action potential onset. For $t>1 \mathrm{~ms}$ data points have been fitted by a double exponential. The spike afterpotential of the specific cell shown in Fig. 1A was fitted by time constants $\tau_{\text {fast }}^{A P}=0.14 \mathrm{~ms}$ and $\tau_{\text {slow }}^{A P}=3.38 \mathrm{~ms}$. Over the ensemble of all 24 cells $\tau_{\text {fast }}^{A P}$ was in the range $[0.01 ; 0.7]$ $\mathrm{ms}\left(\tau_{\text {fast }}^{A P}=0.1 \mathrm{~ms}\right.$ on average $)$ and $\tau_{\text {slow }}^{A P}$ was in the range $[0.4 ; 33.4] \mathrm{ms}$ ( $\tau_{\text {slow }}^{A P}=4.8 \mathrm{~ms}$ on average $)$. The exact values are not critical since several combinations of $\tau_{\text {fast }}^{A P}$ and $\tau_{\text {slow }}^{A P}$ can give similar action potential shapes at the resolution of $0.2 \mathrm{~ms}$ per time step. The filter $\kappa_{\infty}$ (Fig. 1B) is approximated by a sum of two exponential functions. The filter of the specific cell shown in Fig. 1B is fitted by time constants $\tau_{\text {fast }}^{\kappa}=0.3 \mathrm{~ms}$ and $\tau_{\text {slow }}^{\kappa}=7.8 \mathrm{~ms}$. Across cells, the time constants $\tau_{\text {fast }}^{\kappa}$ and $\tau_{\text {slow }}^{\kappa}$ were in the range $[0.01 ; 0.5] \mathrm{ms}$ $\left(\tau_{\text {fast }}^{\kappa}=0.2 \mathrm{~ms}\right.$ on average $)$ and $[1.7 ; 7.8] \mathrm{ms}\left(\tau_{\text {slow }}^{\kappa}=5 \mathrm{~ms}\right.$ on average). The amplitude of $\kappa_{\infty}$ was in the range [0.001; $0.026]{\mathrm{G} \Omega \mathrm{s}^{-1}}^{-1}\left(0.0059 \mathrm{G} \Omega \mathrm{s}^{-1}\right.$ on average $)$. To avoid numerical problems, $\kappa_{\infty}$ was time-shifted so that the peak value is at time $t=0$. The relatively small value of $\tau_{\text {slow }}^{\kappa}$ indicates that the latter time constant cannot be directly identified with the passive membrane time constant at rest. Due to activation of additional conductances during stimulation, notably conductances responsible for spike-frequency adaptation and voltage rectification, the effective time constant during stimulation with a random current is shorter than the expected time constant at resting potential. It is therefore more like a "working" membrane time-constant (Koch et al., 1996 ) or "effective" membrane time constant (Gerstner et al., 1996). With an Integrate-and-Fire model in mind, we would expect $\kappa_{\infty}$ to be best fitted by a single exponential function whereas we find here a double exponential. This double ex- ponential shape can be explained by the coupling between soma and dendrites (Roth and Häusser, 2001). In general, we found that $\kappa_{\infty}$ does not depend on input characteristics in a statistically significant manner over the regime of input parameters considered ( $F$-test with 0.05 significance level).

To finish the mapping of the minimal model to the target neuron, we need to define a suitable threshold. For this kind of model, the classic approach would be to select a constant or a dynamic threshold (i.e. time-dependent threshold) plus an absolute refractory period to avoid continuous firing. A dynamic threshold has been previously reported to yield a better performance across different stimulation regimes (Jolivet et al., 2004). However, cortical neurons exhibit a rich repertoire of ion channels. As a consequence, pyramidal neurons often display spike-frequency adaptation at different time scales (McCormick et al., 1985; Schwindt et al., 1997). It seems natural therefore to include some kind of adaptation in our modeling framework. Following the model recently proposed by La Camera and colleagues (2004), we use an adapting threshold (see Materials and methods). Each time that a spike is emitted by the minimal model, the threshold is increased by a given amount $A_{\vartheta}$ (Schwindt and Crill, 1982; Powers et al., 1999). It then exponentially decays back to a resting value $\vartheta_{0}^{A}$ with a time constant $\tau_{\vartheta}^{A}$ (see Eq. (4)). This formulation can be seen as a standard model of spikefrequency adaptation (Benda and Herz, 2003). Its advantage is that the average threshold depends linearly on the output frequency $f$. Parameters of the model can therefore be obtained by plotting the optimal threshold value $\vartheta_{\text {cst }}$ (obtained under assumption of a constant threshold) as a function of $f$ and fitting by a linear function (see Materials and methods for further details). Figure 1C shows the frequency dependence of $\vartheta_{\text {cst }}$ for a typical cell. We found that a linear fit provides an excellent approximation of the frequency dependence of the threshold. The range of parameters over all cells is $[-62 ;-38] \mathrm{mV}$ for $\vartheta_{0}^{A}\left(\vartheta_{0}^{A}=-50 \mathrm{mV}\right.$ on average $),[2 ; 12] \mathrm{mV}$ for $A_{\vartheta}\left(A_{\vartheta}=7 \mathrm{mV}\right.$ on average $)$ and $[15 ; 71] \mathrm{ms}$ for $\tau_{\vartheta}^{A}\left(\tau_{\vartheta}^{A}=34 \mathrm{~ms}\right.$ on average). To summarize, for each of the 24 cells, we have determined the spike shape $\eta$ and the linear subthreshold filter $\kappa_{\infty}$ as well as parameters of the threshold mechanism $\vartheta_{0}^{A}, A_{\vartheta}$ and $\tau_{\vartheta}^{A}$. 
A
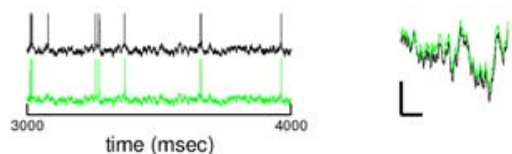

B
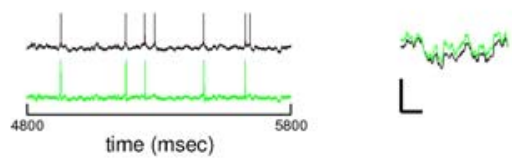

C
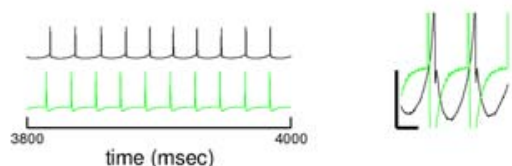

G

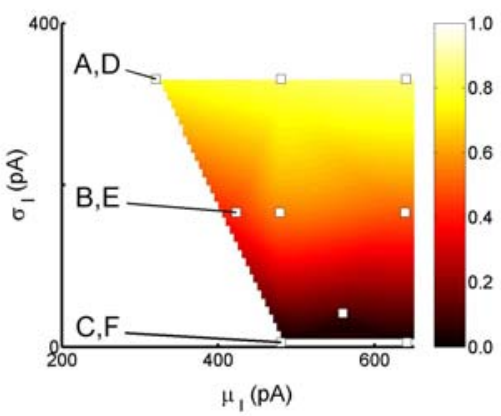

Fig. 2 The predictions of the Spike Response Model (SRM) are compared to electrophysiological measurements. (A). The predicted voltage trace of the SRM (green trace) is compared to the spike train recorded in the target neuron (black trace) when both are driven with the same strongly fluctuating current (highly reliable regime, see text). In the subthreshold regime, predicted membrane voltage and target membrane voltage differ by a few $\mathrm{mV}$ only (inset; vertical bar: $10 \mathrm{mV}$; horizontal bar: $10 \mathrm{~ms}$ ). (B). Same as in $A$ but when the neuron is in the intermediate regime. (C). Same as in $A$ but when the neuron is in the poorly reliable regime (vertical bar: $5 \mathrm{mV}$ ). The inset is not strictly subthreshold regime for clarity reasons. (D). Experimental voltage traces during four repetitions of the same driving current in the highly reliable regime. Inset shows the subthreshold regime (see legend of panel $A$ ). The four voltage traces are nearly indistinguishable. (E). Same as in D but in the intermediate regime. Compare also with the results in panel

To assess the quality of the minimal model, we compare spike trains of the model neuron with the activity of the target neuron. To do this, we use a second set of spike trains that have been set apart and were never touched during model optimization. Both the target neuron and the minimal model are driven with the same realization of a noisy current and corresponding output spike trains are compared on a spikeby-spike basis. We find that the SRM yields a prediction of spike firing times with up to $75 \%$ of spike timing correct (within $\pm 2 \mathrm{~ms}$ ) when the standard deviation of the driving current $\sigma_{I}$ is large $\left(\sigma_{I} \geq 300 \mathrm{pA}\right)$, reasonable predictions in an intermediate region $\left(\sigma_{I} \approx 150 \mathrm{pA}\right)$ and poor predictions when $\sigma_{I}$ is small $\left(\sigma_{I} \leq 100 \mathrm{pA}\right)$. We call these three regimes
D

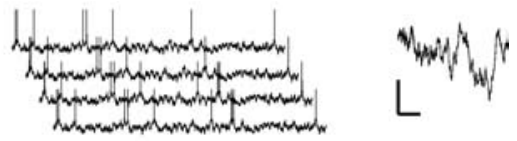

E

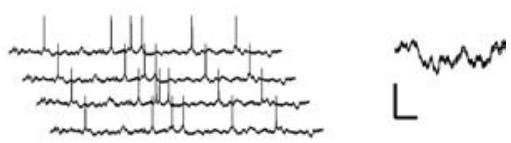

F
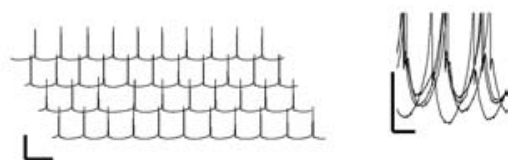

H

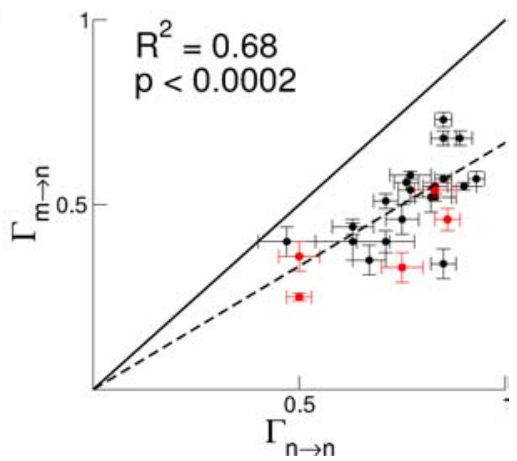

B. (F). Same as in D but in the poorly reliable regime. Compare also with the results in panel C (scale bars: $20 \mathrm{~ms}$ and $50 \mathrm{mV}$; vertical bar: $5 \mathrm{mV}$ ). In panels A-F, action potentials are clipped at $+20 \mathrm{mV}$ except in insets $\mathrm{C}$ and $\mathrm{F}$ where they are clipped at $-40 \mathrm{mV}$. (G). The inter-trial reliability of a typical target neuron in terms of the coincidence factor $\Gamma_{n \rightarrow n}$ plotted in color code as a function of the mean drive $\mu_{I}$ and the standard deviation of the current $\sigma_{I}$ (symbols). The surface is interpolated with cubic splines. $(\mathrm{H})$. The quality of predictions of the SRM $\left(\Gamma_{m \rightarrow n}\right)$ is plotted versus the inter-trial reliability of the target neuron for each data set $\left(\Gamma_{n \rightarrow n}\right)$. The diagonal line constitutes the upper limit of predictions (see text). In each case, the mean (symbols) is plotted with one standard deviation (error bars). On average, the ratio between $\Gamma_{m \rightarrow n}$ and $\Gamma_{n \rightarrow n}$ is 0.65 (dashed line). The values for one given cell are plotted in red for illustrative purpose

highly reliable, intermediate and poorly reliable. Figure $2 \mathrm{~A}-$ $\mathrm{C}$ show the predictions of the SRM compared to the spike train produced by the target neuron for a typical cell in all three regimes. We note that, in both highly reliable and intermediate regimes, spike trains are very similar. Most spikes are predicted with the correct timing although some spikes may be missed or added due to the strict threshold condition for spike emission in the minimal model. Furthermore, the insets show that, in the subthreshold regime, the SRM performs quite well in predicting the membrane voltage. The two traces are almost indistinguishable in the inset of panel A (reliable regime) while one can already see some differences in the inset of panel B (intermediate regime). 
In order to get a quantitative measure of the quality of predictions of the subthreshold voltage, we measured the coefficient of correlation $c$ between the predicted subthreshold voltage and the target subthreshold voltage. The coefficient of correlation $c$ is measured independently for each set of input parameters (i.e. for each couple $\mu_{I}, \sigma_{I}$ ) but including all repetitions for this specific set. We found no statistically significant differences between the different recorded cells (one-way ANOVA and Levene's test for equal variance with 0.05 significance level) while we found significant differences between regimes. The average coefficient of correlation is $c=0.85 \pm 0.08$ (mean \pm s.d.) in the highly reliable regime and $c=0.75 \pm 0.09$ in the intermediate regime. Predictions in the poorly reliable regime are significantly worse. Notably, although the predicted spike train resembles the target spike train (panel $\mathrm{C}$ ), the subthreshold voltage is poorly predicted and $c=0.2 \pm 0.2$.

In order to develop a quantitative reference of the quality of the predictions of firing times, we consider the intrinsic reliability of the target neuron itself. Obviously, if a neuron responded perfectly reliably over several repetitions of the same time-dependent input, it would generate spikes always at the same firing times. A model should then be able to reproduce the same firing times. If, however, a neuron reproduces in the second trial only $50 \%$ of its spike with the same timing (at resolution $\pm 2 \mathrm{~ms}$ ), we cannot expect a model neuron to reproduce more than $50 \%$ of the neuronal spike times. Hence, the intrinsic reliability of a real neuron provides an upper bound for the predictability of spike trains. Several experimenters have studied neuronal reliability (Bryant and Segundo, 1976; Shadlen and Newsome, 1988; Mainen and Sejnowski, 1995; Reich et al., 1997; Kara et al., 2000; Reinagel and Reid, 2002). In particular, Mainen and Sejnowski (1995) have reported that the reliability of cortical neurons can crucially depend on the input scenario. In order to evaluate the intrinsic reliability of the neurons, we repeated in four neurons injection of the same noisy current and recorded the output spike train ( $N=4$ repetitions). Figure 2D-F shows the response of a typical neuron to four repetitions of the same input. Note that the segments in panels D-F correspond to the segments plotted in panels A-C. In both highly reliable and intermediate regimes ( $D$ and $E$ ), spike trains are very similar. Insets show a zoom on the subthreshold regime. Interestingly, we remark that the four traces fall on top of each other in insets of panels D and $E$ where our model yields the best predictions of the subthreshold voltage (compare with insets of panels A and B). The coefficient of correlation $c$ of the subthreshold voltage between repetitions is $c=0.97 \pm 0.05$ (mean \pm s.d.) in both the highly reliable and the intermediate regime. These two regimes are not statistically different (one-way ANOVA and Levene's test for equal variance with 0.05 significance level). In the poorly reliable regime, although repetitions look sim- ilar, precise timing of spikes is not conserved (panel F). The subthreshold voltage is less reliably reproduced across repetitions (inset; $c=0.90 \pm 0.08$ ). Again, we did not find statistically significant differences between the different cells.

We now move to a quantitative estimate of the intrinsic reliability of the target neuron. To do so, we use the coincidence factor $\Gamma$ (Kistler et al., 1997). This quantity gives an estimate of the similarity of two spike trains in terms of the spike timing with some precision $\pm \Delta$. In short, a maximal value of 1 is reached if and only if the two spike trains contain the same number of spikes and with the same timing $( \pm \Delta)$. A value of 0 is reached if the similarity between the spike trains is not better than that between two random spike trains generated by homogeneous Poisson processes at the same rates. $\Gamma$ can be lower than 0 if the two spike trains show anticorrelations (see Eq. (6) for a definition of $\Gamma$ ).

Figure $2 \mathrm{G}$ shows the coincidence factor $\Gamma_{n \rightarrow n}(n \rightarrow n$ is short for neuron compared to itself) computed between responses to repetitions of the same noisy current for a sample neuron. It is plotted versus the mean drive $\mu_{I}$ and the current's standard deviation $\sigma_{I} . \Delta$ is set to $2 \mathrm{~ms}$. We find that the mean drive $\mu_{I}$ has almost no impact on $\Gamma_{n \rightarrow n}$ (measured cross-correlation coefficient $c=0.04 ; p=0.81$ ). On the other hand, $\sigma_{I}$ has a strong impact on the reliability of the neuron $(c=0.93 ; p<0.0001)$. When $\sigma_{I}$ is large $\left(\sigma_{I} \geq 300 \mathrm{pA}\right), \Gamma_{n \rightarrow n}$ reaches a plateau at about $0.84 \pm 0.05$ (mean \pm s.d.). When $\sigma_{I}$ decreases to $100 \leq \sigma_{I} \leq 300 \mathrm{pA}$, $\Gamma_{n \rightarrow n}$ quickly drops to an intermediate value of $0.65 \pm 0.1$ and finally drops down to $0.09 \pm 0.05$ for $\sigma_{I} \leq 100 \mathrm{pA}$. These findings are stable across the different neurons that we recorded and repeat the findings of Mainen and Sejnowski (1995). In the following, we abandon the data of the poorly reliable regime since exact spike timing is not controlled by the stimulus at all (i.e. $\Gamma_{n \rightarrow n} \approx 0$ ). More specifically, we abandon all the data where $\sigma_{I} \leq 150 \mathrm{pA}$ that correspond to $\Gamma_{n \rightarrow n}$ in the range $[0 ; 0.3]$. These data with small input variance $\left(\sigma_{I} \leq 150 \mathrm{pA}\right)$ are also of less importance with respect to in vivo conditions.

We use the intrinsic reliability $\Gamma_{n \rightarrow n}$ as a reference in order to evaluate the quality of our model. Our minimal model is stimulated with the same noisy current as the one used to stimulate the target neuron. We compare the output spike train of the model with all available responses (repetitions) of the target neuron for the same set of $\mu_{I}$ and $\sigma_{I}$ and compute the coincidence factor $\Gamma_{m \rightarrow n}(m \rightarrow n$ is short for model compared to neuron). $\Gamma_{m \rightarrow n}$ is plotted versus $\Gamma_{n \rightarrow n}$ in Fig. $2 \mathrm{H}$ for highly reliable and intermediate regimes for the four cells where trial repetitions of the input were performed. Since the intrinsic reliability of a given cell is lower in the intermediate regime than in the highly reliable regime, the same cell appears with several values of $\Gamma_{n \rightarrow n}$ in the graph. We find that the predictions of our minimal model are close to the natural upper bound set by the intrinsic reliability of the 
neuron. On average, the minimal model achieves a quality $\Gamma_{m \rightarrow n}$ which is $65 \%$ ( $\pm 3 \%$ s.e.m.) of the upper bound, i.e. $\Gamma_{m \rightarrow n}=0.65 \cdot \Gamma_{n \rightarrow n}($ panel H). It reaches a maximal value of about $75 \%$. Furthermore, let us recall that due to the definition of the coincidence factor $\Gamma$, the threshold for statistical significance here is $\Gamma_{m \rightarrow n}=0$. All the points are well above this value, hence highly significant.

These results extend to the cells where no trial repetitions of the input were available $(N=20)$. Although it is not possible to directly compare the coincidence factor $\Gamma_{m \rightarrow n}$ to the intrinsic reliability $\Gamma_{n \rightarrow n}$ of the real neuron, Fig. $3 \mathrm{~A}$ shows that $\Gamma_{m \rightarrow n}$ for these cells is in the same range as the one observed for the cells where trial repetitions of the input were performed (Z-test with 0.01 significance level, see Materials and methods). This is true except for 1 cell (out of 20) where our approach failed and $\Gamma$ was always smaller than 0.25 . This cell was discarded for the rest of our analysis. Finally, we compare the predictions of our minimal model to the target neuron in terms of two other indicators. The first one is the $C_{v}$ of the interspike interval distribution and the second one is the mean output rate. As predicted spike trains are very similar to target spike trains, we expect $C_{v}$ and mean rate of the model spike trains to be similar to those of the target spike trains. Figure 3B shows that this is indeed the case. The $C_{v}$ of the model spike trains roughly corresponds to that of the target trains. Moreover, the mean output rate is very well predicted except in some occasional cases at high frequencies (Fig. 3C). Note that Rauch and colleagues (2003) have reported that an almost perfect prediction of the mean output rate is possible with a very similar approach. However, their model was tuned to predict the mean output rate and not the timing of spikes. Previous model studies had shown that a model with a threshold simpler than the one used here is able to reliably predict the spike train of more detailed neuron models (Jolivet and Gerstner, 2004; Jolivet et al., 2004). Here, we used a threshold including an adaptation mechanism. Without adaptation, i.e. when the sum over all preceding spikes in Eq. (4) is replaced by the contribution of the last emitted spike only, it is still possible to reach the same quality of predictions for each driving regime under the condition that the three threshold parameters $\left(A_{\vartheta}, \vartheta_{0}^{A}\right.$ and $\left.\tau_{\vartheta}^{A}\right)$ are chosen differently for each set of input parameters $\mu_{I}$ and $\sigma_{I}$. In contrast to this, the model with adaptation presented here achieves the same level of predictive quality with one single set of threshold parameters. This illustrates the importance of adaptation in Integrate-and-Fire-type models. The adaptation mechanism adds features well beyond a simpler non-adapting model and greatly improves its generalization capacity.

Finally, the question arises whether our minimal model preferentially predicts intrinsically reliable spikes or not. In order to elucidate this issue, we define a reliability measure for spikes produced by the real neuron. In short, an intrinsic
A

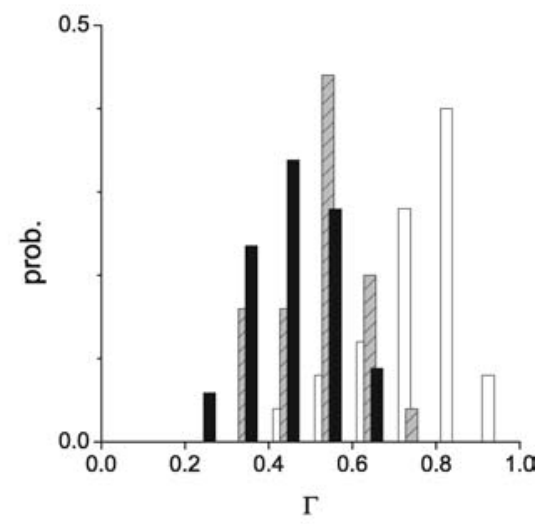

B
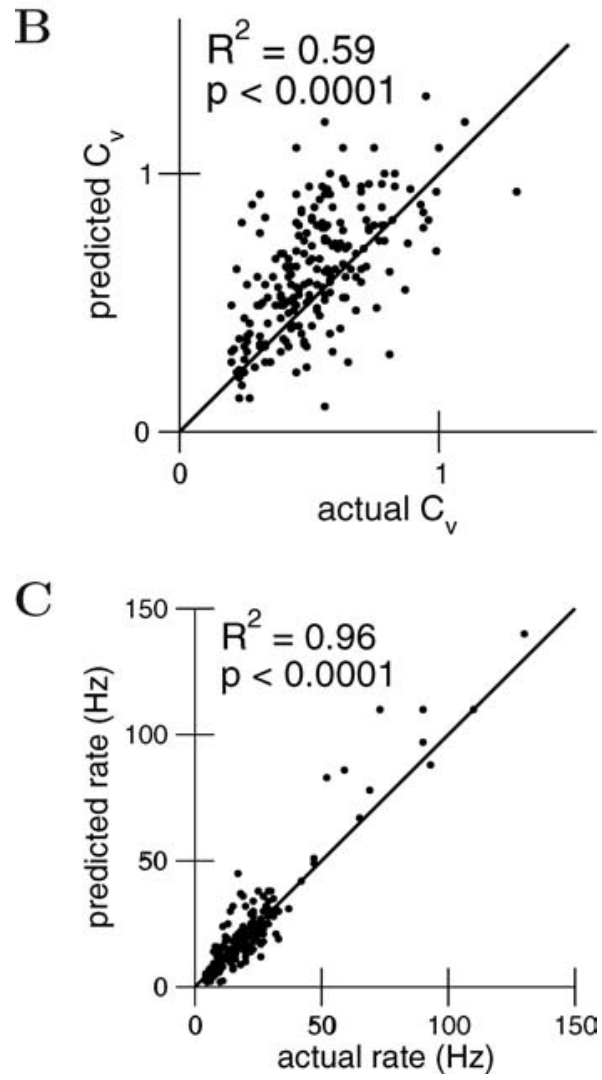

Fig. 3 (A). Comparison of coincidence factor $\Gamma$. The distribution of $\Gamma_{m \rightarrow n}$ for the neurons without repetitions (black) is compared to the distribution of coincidence factor $\Gamma_{m \rightarrow n}$ for the neurons with repetitions (shaded gray) and to the distribution of $\Gamma_{n \rightarrow n}$ for the neurons with repetitions (white). The distribution of $\Gamma_{m \rightarrow n}$ for neurons with repetitions is not significantly different from the distribution of $\Gamma_{m \rightarrow n}$ for neurons without repetitions (Z-test with 0.01 significance level). (B). The $\mathrm{C}_{v}$ of the interspike interval distribution predicted by the model is compared to the real neuron. Each point represents one comparison for a given realization of input current. (C). Same as in B but for the mean firing rate

reliability coefficient is defined for each spike by the fraction of trial repetitions where it appears with the same timing in the data $( \pm 2 \mathrm{~ms})$. It returns a maximal value of 1 only if the spike appears reliably in all repetitions. For example, suppose we observe spikes in trials 1 to 4 at times $t=10 \mathrm{~ms}$ (trial 1$), t=10 \mathrm{~ms}$ (trial 2$), t=8.5 \mathrm{~ms}(\operatorname{trial} 3)$ and $t=11.5 \mathrm{~ms}$ 
A

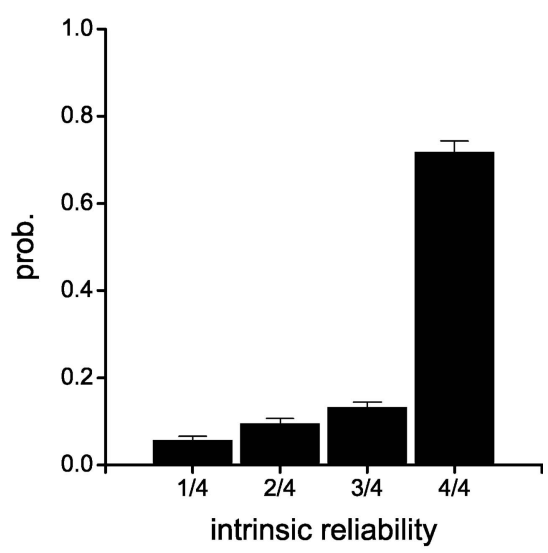

B

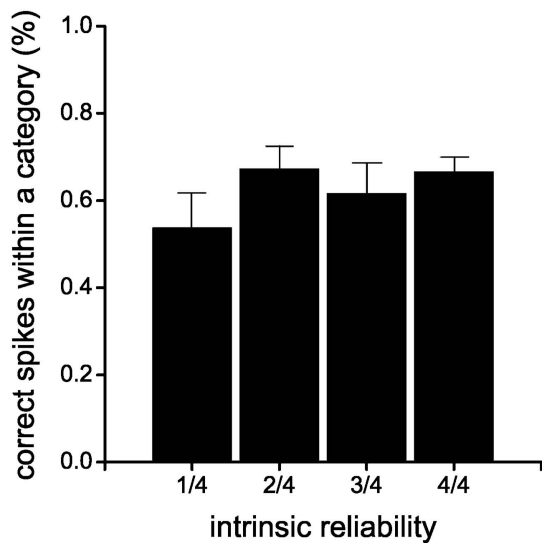

Fig. 4 (A). Repartition of correctly predicted spikes as a function of their intrinsic reliability over 4 repetitions. An intrinsic reliability of 3/4 implies that in 3 out of 4 trials, the cortical neuron generated an action potential at time $t \pm \Delta$ with $\Delta=2 \mathrm{~ms}$. Data are collected for all available recordings of a given cell in intermediate and highly reliable regimes (mean \pm s.e.m.). (B). Percentage of correctly predicted spikes in each category of intrinsic reliability (mean \pm s.e.m.). Same dataset as in (A)

(trial 4). In this case, we would give spikes in trials 1 and 2 a reliability coefficient of $4 / 4$ but spikes in trials 3 and 4 would receive a reliability coefficient of $3 / 4$ since they are distant from each other by more than $2 \mathrm{~ms}$. Figure $4 \mathrm{~A}$ shows that $72 \%$ of the spikes were reproduced in all four repetitions, $13 \%$ in three repetitions, $9 \%$ in two repetitions and $6 \%$ occurred in only one trial. In Fig. 4B, we measure the percentage of correctly predicted spikes in each of the four categories that are present in panel A. We do not find significant differences between categories ( $t$-test with 0.05 significance level). The model correctly predicts $0.63 \pm 0.03$ (mean \pm s.e.m.) of spikes in each category. We will come back to this issue later in this paper. We found no indications that the intrinsic reliability of a spike or the ability of our model to predict a spike is dependent on the length of the precedent interspike interval.

So far, we have proposed a method that allows building a minimal deterministic model for cortical neurons given intracellular recordings. We have found that this model, although very simple, is able to quantitatively predict with the correct timing ( $\pm \Delta=2 \mathrm{~ms}$ ) about $65 \%$ of the spikes that could be predicted considering the limited neuronal reliability. Not only the spikes but also the subthreshold membrane voltage and the output firing rate are most of the time predicted correctly. Finally, the predicted $C_{v}$ of the interspike interval distribution is usually in the correct range as well. However, our model is still deterministic. A stochastic model would be better suited to account for the natural variability of neurons (Stein, 1967; Tuckwell, 1988; Schneidman et al., 1998; Keat et al., 2001; Arcas et al., 2003). In the next subsection, we go further into the study of the variability of neuronal response and turn our deterministic model into a stochastic one by replacing the strict threshold by a firing probability.

\subsection{Spike train variability and stochastic model of neuronal activity}

In the previous subsection, we have shown that a simple deterministic model has significant predictive power for the spike train of cortical cells. However, the reliability of the response of cortical neurons is limited and spikes are sometimes emitted because of intrinsic noise sources. Therefore, it has been argued that the response of neurons is best described by stochastic models (Stein, 1967; Tuckwell, 1988; Arcas et al., 2003). For evaluation of our stochastic model, we use the subset of $N=4$ neurons where every realization of a stimulus with given mean $\mu_{I}$ and standard deviation $\sigma_{I}$ was repeated four times.

In order to gain insight into the mechanisms of spike time unreliability, we first performed spike triggered averaging (STA) and separated spikes on the basis of their reliability. In short, the intrinsic reliability coefficient for each spike is defined as the fraction of trial repetitions where it appears with the same timing ( $\pm 2 \mathrm{~ms}$ ). Since the experimental data contains four trial repetitions, the coefficient can take values $1 / 4,2 / 4,3 / 4$ and $4 / 4$. Figure $5 \mathrm{~A}$ shows the first moment of STAs for voltage (top) and driving current (middle) in a sample spike train for the least (appear in only one trial) and most reliable spikes (appear in all trials). We observe no significant differences between STAs in the range -10 to $0 \mathrm{~ms}$ before the spike. Since there are no differences between STAs for driving current, we cannot expect the deterministic model to show any differences in STA for predicted voltage. Furthermore, we did not find significant differences between the second moments of the STAs before the spike (data not shown). Finally, Fig. 5A (bottom) shows that there are no significant differences between the distributions of the effective spike initiation threshold of least and most reliable spikes. These considerations explain the results of the deterministic model in Fig. 4. Our minimal model only receives the driving current as input and produces spikes on the basis of a strict threshold crossing criterion. As no differences are found in 


\section{A}

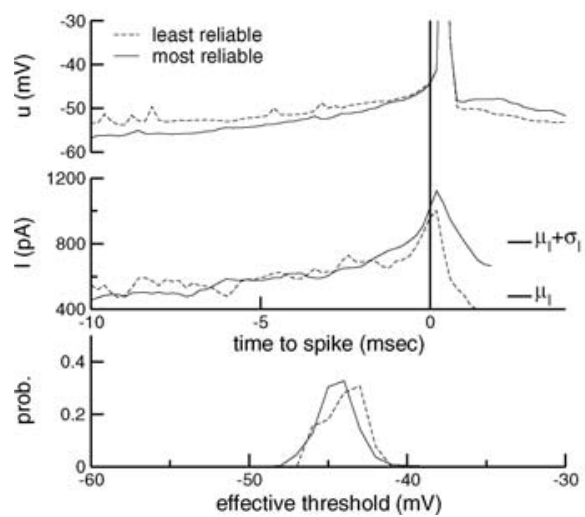

Fig. 5 (A). Spike triggered averaging of the voltage (top) and driving current (middle) calculated separately for the least and most reliable spikes. The distribution of the effective spike threshold as defined by experimental spike detection procedure (see Materials and methods) is compared for the less and most reliable spikes (bottom). (B). Histogram of $(u-\vartheta)$ at real neuron's firing times (black) and the histogram of

STAs for voltage and current between least and most reliable spikes, our minimal model cannot distinguish between them. Therefore, it slightly overestimates the occurrence of unreliable spikes and underestimates that of reliable spikes as shown in Fig. 4B.

We now transform our deterministic model into a stochastic one. There are various ways to introduce noise in a single neuron model (see Gerstner and Kistler (2002) for a review). In the case of our deterministic minimal model, we found that the subthreshold membrane voltage is often well predicted (at least in the highly reliable and intermediate regimes) while it is sometimes difficult to predict the spikes at a correct timing because of the strict threshold condition for spike emission. It seems natural then, to turn the strict threshold condition into a stochastic firing criterion while keeping the rest of the model unchanged. More precisely, the strict condition for spike emission is replaced by a voltage-dependent instantaneous probability of firing $f(u-\vartheta)$. We choose $f(u-\vartheta)=\exp [(u-\vartheta) / \delta u] / \tau_{s}$ with $\vartheta$ the threshold, $\delta u$ the width of the spike trigger zone and $\tau_{s}$ the mean time to spike emission at threshold. To maintain the features of the model of the previous subsection, $\vartheta$ is identified with the adapting threshold introduced above. To evaluate the two other parameters $\tau_{s}$ and $\delta u$, we compute the histogram of $(u-\vartheta)$ at real neuron's firing times and the histogram of $(u-\vartheta)$ at non-firing times using the predictions of our minimal model. By comparing these two histograms, i.e. by taking for each voltage-difference the ratio of the number of instances with action potential triggered to the total number of instances with that voltage, it is possible to estimate the probability of firing in a time bin $d t$ given the voltage $u$ and from this, $\delta u$ and $\tau_{s}$. Fig. 5B shows the two histograms for a sample spike train and the resulting firing
B

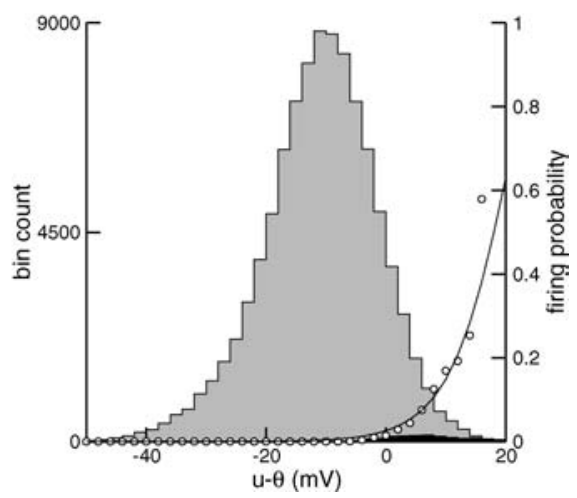

$(u-\vartheta)$ at non-firing times (gray) using the predictions of our minimal model. By comparing these two histograms, i.e. by taking the ratio of the two histograms (see text for further details), gives an estimate of the firing probability in a time bin $d t$ in function of the voltage (circles). It is fitted by the theoretical firing probability given in Eq. (10) in order to extract the parameters of the stochastic model $\tau_{s}$ and $\delta u$ (solid line)

probability. Across all cells, we found $\tau_{s}=19 \pm 13 \mathrm{~ms}$ and $\delta u=4 \pm 1 \mathrm{mV}$ (mean \pm s.d.). This finishes the construction of our stochastic adapting minimal model of neuronal activity.

The first important finding is that the stochastic model improves the performances in the sense that the probability of producing a spike is lowered when the spike is unreliable in the real spike train. Figure 6 shows that the firing probability is significantly lowered for all categories of unreliable spikes while it stays at about the same level for reliable spikes (compare with Fig. 4B). This is likely to be due to the fact that unreliable spikes are elicited by a shorter depolarizing excursion compared to reliable spikes (see Fig. 5A, middle panel, time $>0$ ). This implies that, in case of unreliable spikes, the voltage is only for a short period close to the threshold. Hence, the probability of firing a spike at the same time as the pyramidal cell $\pm \Delta=2 \mathrm{~ms}$ is lower for unreliable spikes than for reliable ones. Finally, Fig. 6 also shows that the probability of firing a spike in an isolated time bin where no spike is present in the real spike train is close to zero.

In order to evaluate whether the stochastic adapting minimal model generates spike trains with the same statistics as the real neuron, we compare the peristimulus time histograms (PSTHs). The PSTH is constructed by counting the number of spikes appearing in each time bin across all repetitions. The resulting vector is then divided by the number of repetitions and by the time step ( $0.2 \mathrm{~ms}$ here). The raw PSTH is smoothed by filtering with a normalized Gaussian window with width $2 \mathrm{~ms}$ (Fig. 7A). The stochastic adapting minimal model is then simulated several times $(N=1000$ repetitions) to accumulate data. A PSTH is constructed for the minimal model in the same way as for the real neuron. PSTHs are then compared to each other and we measure 


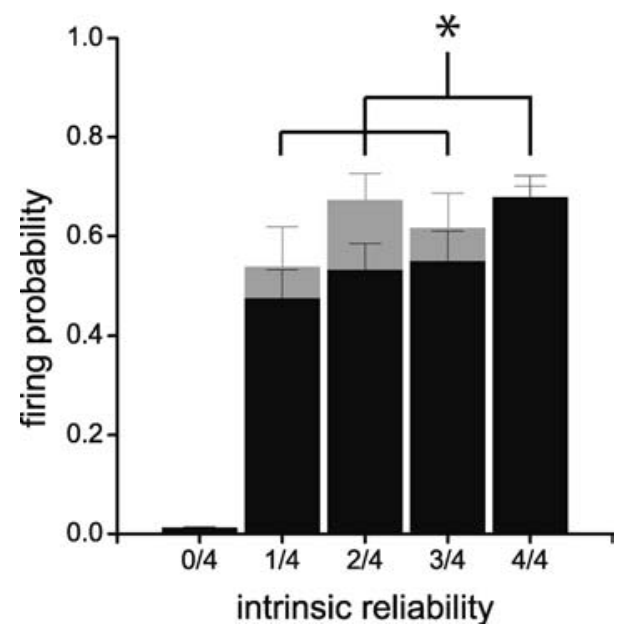

Fig. 6 Firing probability of the minimal model computed at the cell's firing time [ $\pm \Delta=2 \mathrm{~ms}$, see Materials and methods Eq. (8) for further details] plotted versus the intrinsic reliability measured in real spike trains (black). Results of Fig. 3B are repeated in the back for comparison (grey). Firing probability is significantly lower for intrinsic reliability $\leq 3 / 4$ than that for $4 / 4$ (paired $t$-test, ${ }^{*} p=0.001$ )

their similarity by computing the cross-correlation between the two sequences. Figure 7B shows the predictions of the stochastic minimal model. The correlation between the two smoothed histograms $(c=0.83)$ is highly significant. Across all cells, we found that $c=0.74 \pm 0.09$ (mean \pm s.d.).

In summary, we found that our stochastic adapting threshold model accounts for much of the variability of real neurons. PSTHs predicted by the adapting stochastic threshold model are strongly correlated with those of real neurons. In particular, spikes that occur with low reliability (1/4) in the experiments with real neurons are predicted with lower probability by the model than the highly reliable ones. Nevertheless, predictions and PSTHs are not perfect since the probability of highly reliable spikes is systematically underestimated and that of unreliable ones overestimated.

\section{Discussion}

Mapping real neurons to simplified neuronal models has benefited from many developments in recent years (Brillinger, 1988; Kistler et al., 1997; Keat et al., 2001; Arcas et al., 2003; Izhikevich, 2003; Rauch et al., 2003; Jolivet et al., 2004; Paninski et al., 2005) and was applied to both in vitro and in vivo recordings (Brillinger and Segundo, 1979; Stevens and Zador, 1998; Keat et al., 2001; Rauch et al., 2003). The model used in the present paper is identical to the model of Keat et al. (2001), except that noise sources, experimental protocol and fitting procedures are different. Our model is also closely linked to the one of Stevens and Zador (1998) (see Section 4.2.2 of
Gerstner and Kistler 2002) for a discussion). While the model of Brillinger (1988) can also be mapped to our model, his identification technique is optimized to extracellular recordings.

In this paper, we applied our technique of step-by-step parameter estimation of our model neuron to patch-clamp recordings of layer 5 pyramidal neurons of the rat somatosensory cortex recorded in vitro. In the first part of this paper, we find that, even though very simple, our deterministic minimal model yields very good performances in quantitatively predicting neuronal spike trains. Most of the time, the predicted subthreshold membrane voltage differs from the recorded one by a few millivolts only. The mean firing rate of the minimal model corresponds to that of the real neuron. The statistical structure of the spike train is approximately conserved since we observe that the coefficient of variation $\left(C_{v}\right)$ of the interspike interval distribution is predicted in the correct range by our minimal model. But most important, our minimal model has the ability to predict spikes with the correct timing $( \pm 2 \mathrm{~ms})$ and the level of prediction that is reached is close to the intrinsic reliability of the real neuron in terms of spike timing (Mainen and Sejnowski, 1995). This model has two limitations. First, it overestimates slightly the firing probability of unreliable spikes whereas it underestimates the firing probability of highly reliable spikes. This is partly due to the strict deterministic firing criterion. Finally, instead of using a deterministic threshold model, we also considered a noisy threshold model. The noisy threshold model can account for some of the intrinsic unreliability of a neuron. Furthermore, it brings improvements in terms of making a distinction between reliable and unreliable spikes. More precisely, with the stochastic threshold model, we find that the firing probability is lower in cases where spike firing of the cortical neuron was unreliable than at times where the cortical neuron responded reliably.

Several caveats should be kept in mind for an interpretation of our results. First, in our stimulation protocol, we used random current injection rather than a more realistic random conductance protocol (Robinson and Kawai, 1993; Destexhe et al., 2003). In a previous report (Jolivet and Gerstner, 2004), we had checked the consequences of random conductance injection in a Hodgkin-Huxley-type neuron model of an interneuron (Erisir et al., 1999). We found that random conductance injection mainly changes the effective membrane time constant of the neuron and can be accounted for by making the time course of the optimal linear filter $\left(\kappa_{\infty}\right.$ here $)$ depend on the mean input to the neuron. With simulated data, the minimal model reached the same quality level of predictions when driven by random conductance injection (Jolivet and Gerstner, 2004) as the level it reaches when driven by random current injection (Jolivet et al., 2004). Application of our methodology to dynamic-clamp recordings is the next natural step to take and is in progress. 
A
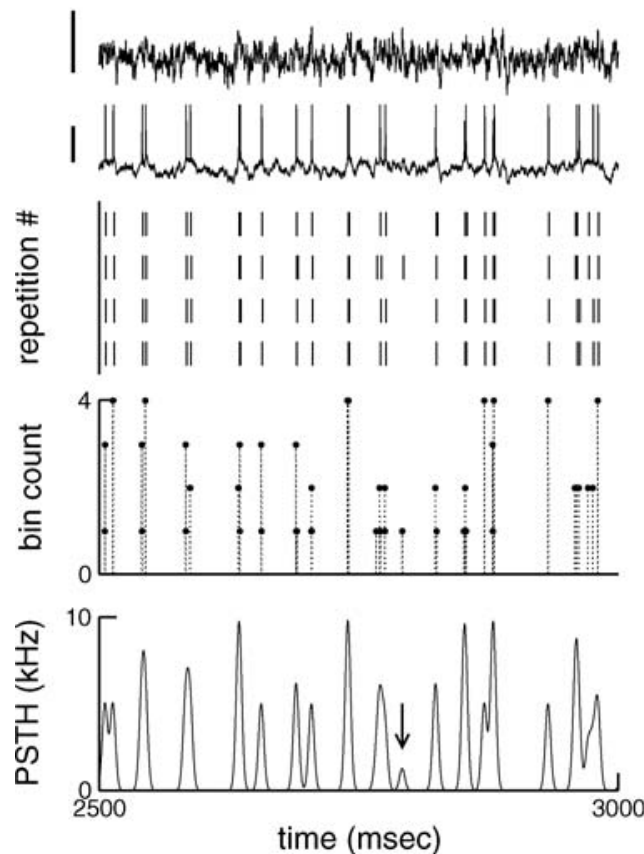

Fig. 7 Comparison between the PSTH of the minimal model and PSTH of the real neuron.(A).From top to bottom:driving current (top; scale bar $2 \mathrm{nA}$ ) and one randomly chosen response of the real neuron (second line; scale bar $50 \mathrm{mV}$ ). Raster plot of all repetitions for the real neuron and bin count (number of spikes per time bin summed across all repetitions). Estimated PSTH for the real neurom (bottom).

Second, a largely fluctuating current generated by a random process can only be seen as a poor approximation to the input a neuron would receive in vivo. Our input has stationary statistics with a spectrum that is close to white (cut-off at $1 \mathrm{kHz}$ ). Thus, it does not include temporal correlations that might occur in the range of 10-100 ms (Bair et al., 2001) or longer (Arieli et al., 1996). Moreover, non-stationary effects caused by external stimulation or short collective bursts of the network activity are not taken into account.

Third, all transient effects have been excluded since neuronal data is analyzed in the adapted state. In particular, a random current injection paradigm is not suitable to probe effects of inhibitory rebound (that would be seen after release of a sustained hyperpolarizing signal) or subthreshold adaptation. In fact, the simple threshold model used in this paper would not be capable to describe these effects, in contrast to other effective models with two variables (Izhikevich, 2004; Brette and Gerstner, 2005).

Finally, our experimental paradigm used somatic current injection. Thus, all dendritic non-linearities, including backpropagating action potentials and dendritic spikes (Stuart and Sakmann, 1994; Helmchen et al., 1999; Larkum et al., 2001; Stuart and Häusser, 2001) are excluded. Understanding the functional role of dendritic nonlinearities firing is an intense field of research (Cash and Yuste, 1988; Polsky et al., 2004).
B

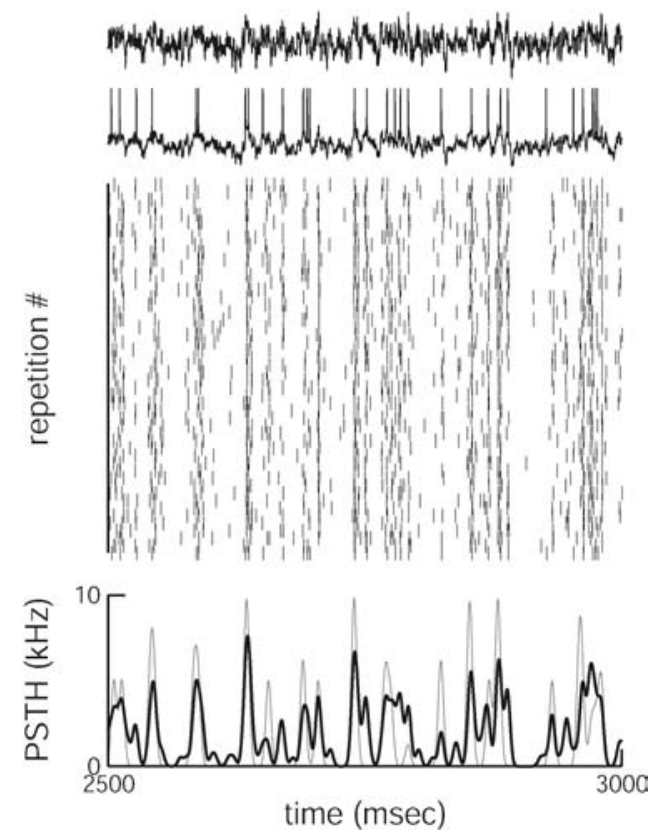

The Gaussian window used to filter the raw PSTH can be seen where a single isolated spike was triggered (arrow).(B). Same as in A but for the minimal stochastic model. The coefficient of correlation measured between the PSTH of the model (thick line) and that of the neuron (thin line, same data as in A) is $c=0.83$

In summary, simple threshold models will never be able to account for all the variety of neuronal responses that can be probed in an artificial laboratory setting. For example, effects of delayed spike initiation due to a pulse that is just superthreshold cannot be reproduced by simple threshold models that combine linear subthreshold behavior with a strict threshold criterion — but could be reproduced by quadratic or exponential integrate-and-fire models (Latham et al., 2000; Fourcaud-Trocmé et al., 2003). However, for random current injection that mimics synaptic bombardment, the picture of a neuron that combines linear summation with a threshold criterion is not too wrong. Moreover, in contrast to more complicated neuron models, the simple threshold model allows rapid parameter extraction from experimental traces; efficient numerical simulation; and rigorous mathematical analysis.

Acknowledgments This work was supported by Swiss National Science Foundation grants number NFN-2100-065268 to WG and number 3100-061335.00 to HRL.

\section{References}

Abeles M (1991) Corticonics. Cambridge, Cambridge University Press. Arcas B, Fairhall A (2003) What causes a neuron to spike? Neural Comp. 15: 1789-1807. 
Arcas B, Fairhall A, Bialek W (2003) Computation in a single neuron: Hodgkin and Huxley revisited. Neural Comp. 15: 1715-1749.

Arieli A, Sterkin A, Grinvald A, A, A (1996) Dynamics of ongoing activity: explanation of the large variability in evoked cortical responses. Science 273: 1868-1871.

Azouz R, Gray C (2000) Dynamic spike threshold reveals a mechanism for synaptic coincidence detection in cortical neurons in vivo. Proc. Natl. Acad. Sci. USA 97: 8110-8115.

Bair W, Koch C (1996) Temporal precision of spike trains in extrastriate cortex of the behaving macaque monkey. Neural Comp. 8: 11851202.

Bair W, Zohary E, Newsome W (2001) Correlated firing in macaque visual area MT: Time scales and relationship to behavior. J. Neurosci. 21: 1676-1697.

Benda J, Herz A (2003) A universal model for spike-frequency adaptation. Neural Comp. 15: 2523-2564.

Berry M, Warland D, Mesiter M (1997) The structure and precision of retinal spike trains. Proc. Natl. Acad. Sci. USA 94: 5411-5416.

Bialek W, Rieke F, de Ruyter van Stevenick R, Warland D (1991) Reading a neural code. Science 252: 1854-1857.

Borg-Graham L, Monier C, Fregnac Y (1998) Visual input evokes transient and strong shunting inhibition in visual cortical neurons. Nature 393: 369-373.

Braitenberg V, Schütz A (1991) Anatomy of the cortex. Berlin, Springer-Verlag.

Brette R, Gerstner W (2005) Adaptive exponential integrate-and-fire model as an effective description of neuronal activity. J. Neurophysiol. 94: 3637-3642.

Brillinger D (1988) The maximum likelihood approach to the identification of neuronal firing systems. Ann. Biomed. Eng. 16: 3-16.

Brillinger D, Segundo J (1979) Empirical examination of the threshold model of neuronal firing. Biol. Cyber. 35: 213-220.

Bryant H, Segundo J (1976) Spike initiation by transmembrane current: a white noise analysis. J. Physiol. 260: 279-314.

Bugmann G, Christodoulou C, Taylor J (1997) Role of temporal integration and fluctuation detection in the highly irregular firing of leaky integrator neuron model with partial reset. Neural Comp. 9: 985-1000.

Buracas G, Zador A, De Weese M, Albright T (1998) Efficient discrimination of temporal patterns by motion-sensitive neurons in primate visual cortex. Neuron 20: 959-969.

Cash S, Yuste R (1988) Input summation by cultured pyramidal neurons is linear and position-independent. J. Neurosci. 18: 10-15.

Cox D, Miller H (1965) The Theory of Stochastic Processes. New-York, Chapman \& Hall.

de Ruyter van Stevenick R, Lowen G, Strong S, Koberle R, Bialek W (1997) Reproducibility and variability in neural spike trains. Science 275: 1805.

De Weese M, Zador A (2003) Binary spiking in auditory Cortex. J. Neurosci. 23: 7940-7949.

De Weese M, Zador A (2004) Shared and private variability in the auditory cortex. J. Neurophysiol. 92: 1840-1855.

Destexhe A, Rudolph M, Paré D (2003) The high-conductance state of neocortical neurons in vivo. Nat. Rev. Neurosci. 4: 739-751.

Diesmann M, Gewaltig M, Aertsen A (1999) Stable propagation of synchronous spiking in cortical neural networks. Nature 402: 529533.

Erisir A, Lau D, Rudy B, Leonard C (1999) Specific K ${ }^{+}$channels are required to sustain high frequency firing in fast-spiking neocortical interneurons. J. Neurophysiol. 82: 2476-2489.

Fourcaud-Trocmé N, Hansel D, van Vreeswijk C, Brunel N (2003) How spike generation mechanisms determine the neuronal response to fluctuating inputs. J. Neurosci. 23: 11628-11640.

Fuortes M, Mantegazzini F (1962) Interpretation of the repetitive firing of nerve cells. J. Gen. Physiol. 45: 1163-1179.

Gawne T, Richmond B (1993) How independent are the messages carried by adjacent inferior temporal cortical neurons. J. Neurosci. 13: $2758-2771$

Gerstner W, Kempter R, van Hemmen J, Wagner H (1996) A neuronal learning rule for sub-millisecond temporal coding. Nature 386: 76-78.

Gerstner W, Kistler W (2002) Spiking Neurons Models: Single Neurons, Populations, Plasticity. Cambridge, Cambridge University Press.

Häusser M, Roth A (1997) Estimating the time course of the excitatory synaptic conductance in neocortical pyramidal cells using a novel voltage jump method. J. Neurosci. 17: 7606-7625.

Heggelund P, Albus K (1978) Response variability and orientation discrimination of single cells in striate cortex of cat. Exp. Brain Res. 32: 197-211.

Helmchen F, Svoboda K, Denk W, Tank D (1999) In vivo dendritic calcium dynamics in deep-layer cortical pyramidal neurons. Nat. Neurosci. 2: 989-996.

Hill A (1936) Excitation and accommodation in nerve. Proc. Roy. Soc. B 119: 305-355.

Ikegaya Y, Aaron G, Cossart R, Aronov D, Lampl I, Ferster D, Yuste R (2004) Synfire chains and cortical songs: temporal modules of cortical activity. Science 304: 559-564.

Izhikevich E (2003) Simple model of spiking neurons. IEEE Trans. Neural Net. 14: 1569-1572.

Izhikevich E (2004) Which model to use for cortical spiking neurons? IEEE Trans. Neural Net. 15: 1063-1070.

Johansson R, Birznieks I (2004) First spikes in ensembles of human tactile afferents code complex spatial fingertip events. Nat. Neurosci. 7: $170-177$.

Jolivet R (2005) Effective minimal threshold models of neuronal activity. $\mathrm{PhD}$ Thesis, Lausanne, Ecole Polytechnique Fédérale de Lausanne (EPFL). http: //icwww.epfl.ch/ rjolivet/publications/reports/PhDthesis.pdf.

Jolivet R, Gerstner W (2004) Predicting spike times of a detailed conductance-based neuron model driven by stochastic spike arrival. J. Physiol.-Paris 98: 442-451.

Jolivet R, Lewis T, Gerstner W (2004) Generalized integrate-and-fire models of neuronal activity approximate spike trains of a detailed model to a high degree of accuracy. J. Neurophysiol. 92: 959-976.

Kara P, Reinagel P, Reid R (2000) Low response variabilities in simultaneously recorded retinal, thalamic, and cortical neurons. Neuron 27: 635-646.

Keat J, Reinagel P, Reid R, Meister M (2001) Predicting every spike: A model for the responses of visual neurons. Neuron 30: 803-817.

Kistler W, Gerstner W, van Hemmen J (1997) Reduction of HodgkinHuxley equations to a single-variable threshold model. Neural Comp. 9: 1015-1045.

Koch C, Bernander O, Douglas R (1995) Do neurons have a voltage or a current threshold for action potential initiation? J. Comp. Neuro. 2: 63-82.

Koch C, Rapp M, Segev I (1996) A brief history of time (constants). Cereb. cortex 6: 93-101.

La Camera G, Rauch A, Lüscher H, Senn W, Fusi S (2004) Minimal models of adapted neuronal response to in vivo-like input currents. Neural Comp. 16: 2101-2124.

Lapicque L (1907) Recherches quantitatives sur l'excitation électrique des nerfs traitée comme une polarization. J. Physiol. Pathol. Gen. 9: 620-635.

Larkum M, Zhu J, Sakmann B (2001) Dendritic mechanisms underlying the coupling of the dendritic with the axonal action potential initiation zone of adult rat layer 5 pyramidal neurons. J. Physiol. 533: 447-466.

Latham P, Richmond B, Nelson P, Nirenberg S (2000) Intrinsic dynamics in neuronal networks. I. Theory. J. Neurophysiol. 83: 808-827.

Lee Y, Schetzen M (1965) Measurement of the wiener kernels of a nonlinear system by cross-correlation. Int. J. Control 2: 237-254. 
Mainen Z, Sejnowski T (1995) Reliability of spike timing in neocortical neurons. Science 268: 1503-1506.

McCormick D, Connors B, Lighthall J, Prince D (1985) Comparative electrophysiology of pyramidal and sparsely stellate neurons of the neocortex. J. Neurophysiol. 54: 782-806.

Paninski L, Pillow J, Simoncelli E (2005) Comparing integrate-andfire models estimated using intracellular and extracellular data. Neurocomp. 65/66: 379-385.

Polsky A, Mel B, Schiller J (2004) Computational subunits in thin dendrites of pyramidal cells. Nat. Neurosci. 7: 621-627.

Powers R, Binder M (1996) Experimental evaluation of input-output models of motoneuron discharges. J. Neurophysiol. 75: 367-379.

Powers R, Sawczuk A, Musick J, Binder M (1999) Multiple mechanisms of spike-frequency adaptation in motoneurones. J. Physiol.Paris 93: 101-114.

Rauch A, La Camera G, Lüscher H, Senn W, Fusi S (2003) Neocortical pyramidal cells respond as integrate-and-fire neurons to in-vivolike input currents. J. Neurophysiol. 90: 1598-1612.

Reich D, Victor J, Knight B, Ozaki T, Kaplan E (1997) Response variability and timing precision of Neuronal Spike trains in-vivo. J. Neurophysiol. 77: 2836-2841.

Reinagel P, Reid R (2002) Precise firing events are conserved across neurons. J. Neurosci. 22: 6837-6841.

Rieke F, Warland D, de Ruyter Van Stevenick R, Bialek W (1996) Spikes-Exploring the neural code. Cambridge, MIT Press.

Robinson H, Kawai N (1993) Injection of digitally synthesized synaptic conductance transients to measure the integrative properties of neurons. J. Neurosci. Meth. 49: 157-165.

Roth A, Häusser M (2001) Compartmental models of rat cerebellar Purkinje cells based on simultaneous somatic and dendritic patchclamp recordings. J. Physiol. 535: 445-472.

Schneidman E, Freedman B, Segev I (1998) Ion channel stochasticity may be critical in determining the reliability and precision of spike timing. Neural Comp. 10: 1679-1703.
Schwindt P, Crill W (1982) Factors influencing motoneuron rhythmic firing: results from a voltage-clamp study. J. Neurophysiol. 48: 875-890.

Schwindt P, O'Brien J, Crill W (1997) Quantitative analysis of firing properties of pyramidal neurons from layer 5 of rat sensorimotor cortex. J. Neurophysiol. 77: 2484-2498.

Shadlen M, Newsome W (1988) The variable discharge of cortical neurons: implications for connectivity, computation, and information coding. J. Neurosci. 18: 3870-3896.

Stein R (1967) Some models of neuronal variability. Biophys. J. 7: 37-68.

Steriade M, Timoveev I, Grenier F (2001) Natural waking and sleep states: a view from inside neocortical neurons. J. Neurophysiol. 85: 1969-1985.

Stevens C, Zador A (1998) Novel integrate-and-fire like model of repetitive firing in cortical neurons. 5th Joint Symposium on Neural Computation, UCSD, La Jolla, CA, Institute for Neural Computation.

Stuart G, Häusser M (2001) Dendritic coincidence detection of EPSPs and action potentials. Nat. Neurosci. 4: 63-71.

Stuart G, Sakmann B (1994) Active propagation of somatic action potentials into neocortical pyramidal cell dendrites. Nature 367: 69-72.

Theunissen F, Miller J (1995) Temporal encoding in nervous systems: a rigorous definition. J. Comp. Neuro. 2: 149-162.

Troyer T, Miller K (1997) Physiological gain leads to high ISI variability in a simple model of a cortical regular spiking cell. Neural Comp. 9: $971-983$.

Tuckwell H (1988) Introduction to Theoretic Neurobiology. Cambridge, Cambridge University Press.

Wehr M, Zador A (2003) Balanced inhibition underlies tuning and sharpens spike timing in auditory cortex. Nature 426: 442-446.

Wiener N (1958) Nonlinear Problems in Random Theory. Cambridge, MIT Press. 